\section{Mortalidade infantil pós-neonatal evitável: o que revelam os óbitos em domicílio}

\section{Post neonatal avoidable deaths: what the household deaths reveal}

\author{
Antônio P. Caldeira 1 \\ Elizabeth França 2 \\ Eugênio A. M. Goulart 3
}

1 Departamento de Saúde da Mulher e da Criança. Faculdade de Medicina da Universidade Estadual de Montes Claros. Av. Rui Braga, s. n. Vila Mauricéia. Montes Claros, MG, Brasil. CEP: 39.401-089.

2 Departamento de Medicina Preventiva e Social. Faculdade de Medicina da Universidade Federal de Minas Gerais.

3 Departamento de Pediatria. Faculdade de Medicina da

Universidade Federal de Minas Gerais.

\section{Abstract}

Objectives: to analyze household post neonatal infant mortality occurring in the metropolitan area of Belo Horizonte, Minas Gerais.

Methods: post neonatal deaths due to diarrhea, pneumonia and malnutrition were selected between May 1991 and April 1992. Following hospital investigation, 511 deaths were identified and 396 were included in the study. It was noted that 34,3\% of infant deaths occurred out of the hospital. After hospital investigation and interviews with the children's families, household deaths were compared to those occurring in hospitals.

Results: comparisons indicated that both groups were similar in terms of socioeconomic variables, gestation, childbirth conditions and health status. The multivariate analysis indicated that only the number of health services visited in the last 15 days was associated to place of death, with a 2,5 times risk of dying out of the hospital for children whose families procured less than three healthcare facilities $(O R=$ 2,53). Medical assistance, provided by medical services two weeks prior to death showed that inadequate clinical management was also associated to household death $(O R=1,78)$.

Conclusions: household infant deaths were associated to the low quality medical services provided by healthcare facilities.

Key words Infant mortality, Quality of health care, Services evaluation.

\section{Resumo}

Objetivos: estudar óbitos pós-neonatais ocorridos em domicílio na Região Metropolitana de Belo Horizonte.

Métodos: foram identificados óbitos pósneonatais por diarréia, pneumonia e desnutrição entre maio de 1991 e abril de 1992. Selecionaram-se 511 óbitos após investigação hospitalar, tendo sido estudados 396. Observou-se que 34,3\% desses óbitos ocorreram fora do ambiente hospitalar. Após investigação hospitalar e entrevistas domiciliares, os óbitos domiciliares foram comparados com os óbitos ocorridos em hospitais.

Resultados: a comparação entre os dois grupos de óbitos mostrou semelhança em relação às variáveis socioeconômicas, ambientais, relativas à gestação e parto e relativas à saúde da criança. $\mathrm{Na}$ análise multivariada apenas o número de serviços procurados durante a doença que levou ao óbito esteve associado ao local de ocorrência do óbito, com chance 2,5 vezes maior de morrer em domicílio para as crianças cujas famílias procuraram menos de três serviços de saúde nos 15 dias antecedentes ao óbito $(O R=2,53)$. A assistência especificamente recebida pelas crianças, nas últimas duas semanas anteriores ao óbito, mostrou uma associação significativa entre óbito domiciliar e conduta clínica inadequada $(O R=$ 1,78).

Conclusões: a ocorrência de óbitos infantis em domicílio está associada a uma baixa efetividade dos serviços de saúde.

Palavras-chave Mortalidade infantil, Qualidade dos cuidados de saúde, Avaliação dos serviços 


\section{Introdução}

As estatísticas de mortalidade representam, de modo geral, uma importante fonte de informações epidemiológicas. Entre os diversos índices, a taxa de mortalidade infantil é um dos indicadores mais empregados para a avaliação das condições de vida da população. ${ }^{1}$ Entende-se o óbito infantil como um evento indesejável cujos determinantes encontramse, em sua maioria, fortemente atrelados à estrutura social e econômica do país. 2 Ainda que nas últimas décadas tenha sido verificada uma importante queda nos índices de mortalidade infantil em todo o mundo, sem uma significativa melhora da qualidade de vida, a taxa de mortalidade infantil continua a refletir as marcantes disparidades entre os países desenvolvidos e em desenvolvimento. ${ }^{3}$

O Brasil, apesar das taxas decrescentes, ainda conta com níveis alarmantes e eticamente inaceitáveis de mortalidade infantil. Estudos recentes têm apontado a importância de intervenções múltiplas na redução da mortalidade infantil em todo o mundo, com destaque especial para as ações dos serviços de saúde. ${ }^{4}$ Também tem sido registrada a participação qualitativa e organizacional dos serviços de saúde na determinação do óbito infantil.5,6 Infelizmente, as estatísticas oficiais mostramse muitas vezes incapazes de revelar a verdadeira dimensão do problema, devido ao sub-registro e baixa confiabilidade das mesmas. ${ }^{7-10}$

Um dado particularmente surpreendente identificado em alguns estudos de base populacional tem sido o elevado número de óbitos ocorridos em domicílio: $23 \%$ dos óbitos pós-neonatais no Rio de Janeiro, em 1986,8 44,8\% dos óbitos por pneumonia em menores de cinco anos em pesquisa realizada no sul do país 11 e $18,9 \%$ dos óbitos infantis, segundo estudo de Menezes et al.10 As estatísticas oficiais não conseguem identificar tal realidade, já que muitas vezes, ainda que o óbito tenha ocorrido fora do ambiente hospitalar, a declaração de óbito não revela este fato. ${ }^{8}$ Embora os óbitos domiciliares possam representar um importante indicador de acesso aos serviços de saúde, ou mesmo um evento "sentinela" para a avaliação dos serviços de saúde, 8 essa questão tem sido relativamente pouco abordada na literatura.

A surpreendente proporção de mais de um terço dos óbitos que ocorreu fora do ambiente hospitalar na Região Metropolitana de Belo Horizonte12 remete a um amplo questionamento sobre a acessibilidade dos serviços de saúde e a qualidade da assistência, já que, pelo menos sob o aspecto numérico, a região estudada já possuía na época do estudo uma grande rede pública de centros de assistência ambulatorial contando ainda com 15 hospitais públicos e 50 hospitais privados integrantes do Sistema Único de Saúde (SUS).

A investigação dos óbitos infantis ocorridos fora do ambiente hospitalar compreende a identificação e utilização de um indicador de acessibilidade aos serviços de saúde, mas também disponibiliza dados para discussão sobre a qualidade da assistência à saúde da criança. Em estudo realizado no Rio de Janeiro, as autoras identificaram uma elevada proporção de óbitos em domicílio após atendimento em serviços de saúde. 8 Não se pode deixar de questionar a qualidade desse tipo de atendimento. Considerando-se o grupamento de causas diarréia-pneumoniadesnutrição, aqui estudado, como indicador ou marcador de pobreza, o estudo que ora se apresenta também propõe a reflexão sobre a assistência à saúde para um grupo específico da população.

Mesmo declinante, a mortalidade infantil ainda representa um grande desafio para a saúde pública em Minas Gerais e no Brasil. Particularmente para a Região Metropolitana de Belo Horizonte a mortalidade pós-neonatal era cerca de 16,5 por 1.000 nascidos vivos em 1990,12 ou seja, ainda muito elevada em relação aos países desenvolvidos ou mesmo em relação a alguns países vizinhos da América Latina. Conhecer melhor os determinantes do óbito infantil por diarréia, pneumonia e desnutrição representa uma possibilidade maior de intervenção sobre esses óbitos precoces. Antecipar a participação ativa dos serviços de saúde sobre esse grupamento de causas representa um compromisso ético. Neste trabalho, a partir de uma análise comparativa entre o grupo de óbitos domiciliares e hospitalares, procurou-se identificar possíveis fatores associados à ocorrência do óbito fora do hospital por patologias consideradas causas evitáveis do óbito infantil.

\section{Métodos}

O presente trabalho faz uma abordagem do tema a partir de dados coletados na Região Metropolitana de Belo Horizonte, (RMBA) MG, Brasil, por ocasião da realização de pesquisa de base populacional sobre determinantes do óbito infantil pós-neonatal por diarréia, pneumonia e desnutrição. 12

A área de abrangência deste estudo foi parte da RMBH representada por 13 municípios, além da capital e compreendendo uma população total de aproximadamente 3.360 .000 habitantes.

Foram coletadas, de forma prospectiva, todas as declarações de óbitos pós-neonatais referentes a cri- 
anças residentes na área estudada no período de maio de 1991 a abril de 1992. Inicialmente selecionaramse para investigação todas as declarações de óbito com citação de diarréia, pneumonia ou desnutrição, além de outras com citações que, após investigação, pudessem se enquadrar no critério de caso (anemias, septicemia, desidratação, etc.). Nessa etapa, implantou-se uma busca ativa semanal de óbitos infantis na Secretaria de Estado de Planejamento de Minas Gerais, responsável pela coleta dos óbitos registrados em cartórios. Para cada óbito selecionado, realizou-se uma investigação hospitalar (revisão de prontuários) e/ou domiciliar (entrevista com a família). A definição da causa básica do óbito foi realizada por médicos, segundo as normas padronizadas da nona revisão da Classificação Internacional de Doenças (CID). Foram excluídos os óbitos associados a malformações congênitas importantes, paralisia cerebral, causas perinatais ou prematuridade extrema e os óbitos cuja investigação hospitalar e domiciliar mostraram-se inconclusivas (isto é, quando não foi possível afastar a possibilidade de outras patologias associadas serem determinantes do óbito).

No questionário domiciliar foram levantadas informações relativas às condições socioeconômicas e relacionadas à família, condições ambientais e relacionadas à moradia, condições de gestação e parto, condições de saúde da criança e evolução do processo de doença que gerou o óbito. Priorizou-se a entrevista com a mãe $(95,7 \%$ das entrevistas teve a mãe como respondente), que foi realizada cerca de dois meses, em média, após o óbito. Na investigação hospitalar foram coletadas informações relativas às condições clínicas da criança e procedimentos adotados. Maiores detalhes da metodologia podem ser encontrados em publicação anterior. 13

No presente estudo, foram comparados os óbitos segundo o local de ocorrência do evento. Os critérios utilizados na classificação do óbito como de ocorrência domiciliar foram: registro na declaração de óbito, registro no prontuário hospitalar de óbito a caminho ou no domicílio, relato da mãe, através da entrevista, de que o óbito teria ocorrido fora do ambiente hospitalar, história de óbito em setor de urgência ou no posto de saúde e história de óbito no hospital até duas horas após a internação. Foram classificados como óbitos hospitalares aqueles ocorridos em ambiente hospitalar, com permanência hospitalar, após admissão, superior a duas horas. Foram considerados como não localizados os prontuários não encontrados em pelo menos duas visitas hospitalares e os endereços dos familiares não foram confirmados em pelo menos duas tentativas de localização (ou quando confirmados, não se tenha localizado a família para a entrevista).

Para acesso aos prontuários, foram obtidos previamente apoio e consentimento dos hospitais, assegurando-se total sigilo das informações coletadas. Para a entrevista domiciliar, solicitou-se consentimento formal da família, através de carta de apresentação dos entrevistadores, assegurando-se, igualmente, a confidencialidade das informações.

Além do estudo de variáveis socioeconômicas e das relacionadas às condições maternas e de saúde da criança, uma análise particular foi realizada para os atendimentos prestados às crianças em serviços de saúde nos últimos 15 dias. Procurou-se assim, analisar toda a trajetória da criança durante a patologia que levou ao óbito, como número de serviços procurados, tipo de serviços, intervalo entre os mesmos, tipo de profissional que prestou o atendimento, conduta adotada e aderência à mesma. A avaliação da conduta adotada foi realizada para todos os atendimentos. Foram classificadas como condutas inadequadas: a) a falta de prescrição; b) a prescrição isolada, sem orientação para retorno; c) o uso isolado de medicamentos sintomáticos no local do atendimento, sem prescrições posteriores ou orientações.

As análises estatísticas foram realizadas pelos programas Epi-info ${ }^{14}$ e Multlr. ${ }^{15}$ Para a verificação de associação entre as variáveis e a ocorrência do óbito domiciliar, utilizou-se o teste do qui-quadrado nas tabelas de contingência e o odds ratio (OR) e seu intervalo de confiança a 95\% (IC95\%) como medida da magnitude das associações. A análise multivariada foi realizada através do modelo de regressão logística não condicional. Inicialmente aplicou-se o modelo de regressão por grupamento de variáveis, incluindo-se no modelo todas as variáveis que se mostraram significantes ao nível de $p<0,20$. Numa segunda etapa, todas as variáveis significantes foram incluídas em um mesmo modelo. Admitiu-se, para o modelo final, um nível de significância de 5\%.

Foram inicialmente selecionados 665 óbitos cuja causa básica incluía diarréia, pneumonia ou desnutrição e outras patologias que após investigação pudessem incluir tais causas (anemias, septicemias, etc). Após investigação hospitalar, foram excluídos 154 óbitos. Cerca de $23 \%$ das exclusões foram devidas à definição de outras patologias como causa básica do óbito $(\mathrm{n}=35)$. Também foram excluídos casos associados a malformações congênitas importantes $(n=32)$ e aqueles que apresentavam história clínica inconclusiva $(n=47)$. Excluiram-se ainda os óbitos referentes a um dos irmãos nascidos de partos gemelares $(n=6)$ e crianças institucionalizadas ou não residentes na RMBH ou cuja seleção inicial foi incorreta $(\mathrm{n}=34)$. Foram selecionados, portanto, 511 
óbitos, mas a investigação hospitalar e domiciliar só foi concluída para 396 casos, pois não se conseguiu encontrar a família de 115 crianças após, pelo menos, duas tentativas. Os principais motivos foram: mudança de endereço, endereço inexistente e endereço fornecido de outra família. Esses óbitos foram considerados como perdas da investigação domiciliar (22,5\% dos óbitos selecionados).

\section{Resultados}

Dos 396 óbitos analisados, 9,8\% $(\mathrm{n}=39)$ foram classificados como de ocorrência domiciliar segundo a declaração de óbito. Entretanto, após investigação hospitalar e entrevista com a família, verificou-se que $34,3 \%$ dos óbitos $(n=136)$ eram de ocorrência domiciliar, segundo os critérios adotados no presente trabalho: registro na declaração de óbito $(n=39)$, registro no prontuário hospitalar de óbito a caminho ou no domicílio $(\mathrm{n}=33)$, relato da mãe, através da entrevista, de que o óbito teria ocorrido fora do ambiente hospitalar $(n=43)$, história de óbito em setor de urgência ou no posto de saúde $(\mathrm{n}=13)$ ou história de óbito no hospital até duas horas após a internação $(\mathrm{n}=8)$. Outros 247 óbitos foram classificados como de ocorrência hospitalar e 13 óbitos não puderam ter definição precisa e confiável do local de ocorrência do fato. $\mathrm{O}$ grupo dos óbitos domiciliares apresentava uma idade média de 5,1 meses e as causas identificadas dos óbitos após entrevista domiciliar apresentaram a seguinte distribuição: as pneumonias foram as mais comuns, com frequiência de $59,6 \%(\mathrm{n}=81)$, seguindo-se a desnutrição com $22,8 \%(\mathrm{n}=31)$ e as diarréias com $17,6 \%(n=24)$. Os óbitos hospitalares apresentavam uma idade média de 5,2 meses e o predomínio da desnutrição entre as causas do óbito, com freqüência de $38,1 \%(n=94)$, seguindo-se as pneumonias, com $33,2 \%(\mathrm{n}=82)$ e as diarréias, com $28,7 \%(\mathrm{n}=71)$. Nos dois grupos havia predomínio do sexo masculino (62,5\% dos óbitos domiciliares e $56 \%$ dos óbitos hospitalares).

A análise da situação socioeconômica não mostrou diferença significativa entre os dois grupos de crianças (Tabela 1). A maioria das famílias residia em favelas, em construções precárias, sem rede pública de esgotos ou coleta de lixo. A escolaridade e a situação conjugal da mãe também se mostraram semelhantes em ambos os grupos. As variáveis comportamentais analisadas não mostraram diferenças, destacando-se a elevada proporção de fumantes em ambos os grupos. 
Análise univariada da associação entre variáveis socioeconômicas e ambientais e local de ocorrência do óbito. Região Metropolitana de Belo Horizonte, MG, Brasil, 1992 a 1992.

\begin{tabular}{|c|c|c|c|c|c|}
\hline \multirow{2}{*}{ Variáveis } & \multicolumn{2}{|c|}{ Óbitos domiciliares } & \multicolumn{2}{|c|}{ Óbitos hospitalares } & \multirow{2}{*}{ OR (IC95\%) } \\
\hline & $\mathrm{n}$ & $\%$ & $n$ & $\%$ & \\
\hline \multicolumn{6}{|l|}{ Escolaridade materna } \\
\hline Até três anos & 65 & 47,8 & 119 & 48,2 & $0,98(0,63-1,53)$ \\
\hline Quatro anos ou mais & 71 & 52,2 & 128 & 51,8 & $\begin{array}{l}1,0 \\
(p=0,972)\end{array}$ \\
\hline \multicolumn{6}{|l|}{ Situação conjugal da mãe } \\
\hline Solteira & 25 & 18,4 & 64 & 25,9 & $0,64(0,37-1,11)$ \\
\hline Casada & 111 & 81,6 & 183 & 74,1 & $\begin{array}{l}1,0 \\
(p=0,123)\end{array}$ \\
\hline \multicolumn{6}{|l|}{ Localização da casa } \\
\hline Favelas & 83 & 61,0 & 137 & 55,5 & $1,26(0,80-1,97)$ \\
\hline Bairros/Outros & 53 & 39,0 & 110 & 44,5 & $\begin{array}{l}1,0 \\
(p=0,344)\end{array}$ \\
\hline \multicolumn{6}{|l|}{ Tipo de construção } \\
\hline Precária & 72 & 52,9 & 133 & 53,8 & $0,96(0,62-1,50)$ \\
\hline Adequada & 64 & 47,1 & 114 & 46,2 & $\begin{array}{l}1,0 \\
(p=0,950)\end{array}$ \\
\hline \multicolumn{6}{|l|}{ Moradores/cômodo } \\
\hline 2,0 ou mais & 68 & 50,0 & 148 & 59,9 & $0,67(0,43-1,04)$ \\
\hline Até 2,0 & 68 & 50,0 & 99 & 40,1 & $\begin{array}{l}1,0 \\
(p=0,077)\end{array}$ \\
\hline \multicolumn{6}{|l|}{ Fumantes na casa } \\
\hline Sim & 95 & 69,9 & 194 & 78,5 & $0,63(0,38-1,05)$ \\
\hline Não & 41 & 30,1 & 53 & 21,5 & $\begin{array}{l}1,0 \\
(p=0,077)\end{array}$ \\
\hline \multicolumn{6}{|l|}{ Eletrodomésticos } \\
\hline Até dois diferentes & 53 & 39,0 & 85 & 34,4 & $1,22(0,77-1,92)$ \\
\hline Três ou mais diferentes & 83 & 61,0 & 162 & 65,6 & $\begin{array}{l}1,0 \\
(p=0,437)\end{array}$ \\
\hline \multicolumn{6}{|l|}{ Água para beber } \\
\hline "In natura" & 58 & 42,6 & 85 & 34,4 & $1,42(0,90-2,23)$ \\
\hline Fervida ou filtrada & 78 & 57,4 & 162 & 65,6 & $\begin{array}{l}1,0 \\
(p=0,138)\end{array}$ \\
\hline \multicolumn{6}{|l|}{ Rede de esgotos } \\
\hline Fossa/rua/quintal & 85 & 62,5 & 172 & 69,6 & $0,73(0,46-1,16)$ \\
\hline Rede geral & 51 & 37,5 & 75 & 30,4 & $\begin{array}{l}1,0 \\
(p=0,191)\end{array}$ \\
\hline \multicolumn{6}{|l|}{ Coleta pública do lixo } \\
\hline Não & 94 & 69,1 & 164 & 66,4 & $1,13(0,71-1,82)$ \\
\hline Sim & 42 & 30,9 & 83 & 33,6 & $\begin{array}{l}1,0 \\
(p=0,667)\end{array}$ \\
\hline
\end{tabular}


Em relação às variáveis relacionadas às condições de gestação e antecedentes obstétricos da mãe (Tabela 2), não foram observadas diferenças significantes entre os dois grupos. Destacam-se para ambos os grupos a pequena proporção de mães que fizeram o mínimo de seis consultas pré-natais e a elevada proporção de crianças com baixo peso de nascimento.

Tabela 2

Análise univariada da associação entre variáveis relacionadas às condições maternas e obstétricas e local de ocorrência do óbito. Região Metropolitana de Belo Horizonte, MG, Brasil, de 1991 a 1992.

\begin{tabular}{|c|c|c|c|c|c|}
\hline \multirow{2}{*}{ Variáveis } & \multicolumn{2}{|c|}{ Óbitos domiciliares } & \multicolumn{2}{|c|}{ Óbitos hospitalares } & \multirow{2}{*}{ OR (IC95\%) } \\
\hline & $\mathrm{n}$ & $\%$ & $\mathrm{n}$ & $\%$ & \\
\hline \multicolumn{6}{|l|}{ Idade da mãe } \\
\hline Até 19 anos & 25 & 18,4 & 39 & 15,8 & $1,20(0,67-2,16)$ \\
\hline 20 ou mais & 111 & 81,6 & 208 & 84,2 & $\begin{array}{l}1,0 \\
(p=0,612)\end{array}$ \\
\hline \multicolumn{6}{|l|}{ Idade primeira gravidez } \\
\hline Menos de 16 anos & 24 & 17,6 & 30 & 12,3 & $1,52(0,82-2,83)$ \\
\hline 16 anos ou mais & 112 & 82,4 & 213 & 87,7 & $\begin{array}{l}1,0 \\
(p=0,207)\end{array}$ \\
\hline \multicolumn{6}{|l|}{ Número de gestações } \\
\hline Mais de três & 53 & 39,0 & 96 & 39,3 & $0,98(0,63-1,55)$ \\
\hline Até três & 83 & 61,0 & 148 & 60,7 & $\begin{array}{l}1,0 \\
(p=0,970)\end{array}$ \\
\hline \multicolumn{6}{|l|}{ Abortos prévios } \\
\hline $\operatorname{Sim}$ & 27 & 20,0 & 63 & 25,9 & $0,71(0,42-1,22)$ \\
\hline Não & 108 & 80,0 & 180 & 74,1 & $\begin{array}{l}1,0 \\
(p=0,242)\end{array}$ \\
\hline \multicolumn{6}{|l|}{ Consultas pré-natais } \\
\hline Até cinco & 75 & 56,0 & 146 & 60,6 & $0,83(0,53-1,30)$ \\
\hline Seis ou mais & 59 & 44,0 & 95 & 39,4 & $\begin{array}{l}1,0 \\
(p=0,447)\end{array}$ \\
\hline \multicolumn{6}{|l|}{ Tabagismo na gravidez } \\
\hline Sim & 60 & 44,4 & 107 & 44,4 & $1,00(0,64-1,57)$ \\
\hline Não & 75 & 55,6 & 134 & 55,6 & $\begin{array}{l}1,0 \\
(p=0,921)\end{array}$ \\
\hline \multicolumn{6}{|l|}{ Anemia na gravidez } \\
\hline Sim & 57 & 42,5 & 114 & 47,7 & $0,81(0,52-1,27)$ \\
\hline Não & 77 & 57,5 & 125 & 52,3 & $\begin{array}{l}1,0 \\
(p=0,394)\end{array}$ \\
\hline \multicolumn{6}{|l|}{ Hipertensão na gravidez } \\
\hline Sim & 48 & 35,6 & 68 & 28,6 & $1,38(0,86-2,22)$ \\
\hline Não & 87 & 64,4 & 170 & 71,4 & $\begin{array}{l}1,0 \\
(p=0,199)\end{array}$ \\
\hline \multicolumn{6}{|l|}{ Tipo de parto } \\
\hline Cesariana & 32 & 23,7 & 55 & 22,4 & $1,07(0,63-1,82)$ \\
\hline Normal & 103 & 76,3 & 190 & 77,6 & $\begin{array}{l}1,0 \\
(p=0,880)\end{array}$ \\
\hline \multicolumn{6}{|l|}{ Peso ao nascer } \\
\hline Menor que $2.500 \mathrm{~g}$ & 48 & 35,6 & 75 & 30,7 & $1,24(0,78-1,99)$ \\
\hline $2.500 \mathrm{~g}$ ou mais & 87 & 64,4 & 169 & 69,3 & $\begin{array}{l}1,0 \\
(p=0,398)\end{array}$ \\
\hline
\end{tabular}


A Tabela 3 apresenta as variáveis relacionadas às condições de saúde das crianças. Antecedentes de internação, desidratação e pneumonia mostraram-se como fatores associados ao óbito hospitalar. Também o número de serviços procurados nos últimos 15 dias esteve significantemente associado ao local do óbito. No modelo final da análise multivariada apenas essa variável manteve-se significante com uma chance 2,5 vezes maior de ocorrência de óbito no domicílio para as crianças que tiveram o número de visitas a serviços de saúde igual ou inferior a dois.

Tabela 3

Análise univarida da associação entre variáveis relacionadas às condições de saúde da criança e local de ocorrência do óbito. Região Metropolitana de Belo Horizonte, MG, Brasil, de 1991 a 1992.

\begin{tabular}{|c|c|c|c|c|c|}
\hline \multirow{2}{*}{ Variáveis } & \multicolumn{2}{|c|}{ Óbitos domiciliares } & \multicolumn{2}{|c|}{ Óbitos hospitalares } & \multirow{2}{*}{ OR (IC95\%) } \\
\hline & $\mathrm{n}$ & $\%$ & $n$ & $\%$ & \\
\hline \multicolumn{6}{|l|}{ Amamentação predominante } \\
\hline$<30$ dias & 67 & 49,3 & 109 & 44,5 & $1,21(0,78-1,89)$ \\
\hline \multirow[t]{2}{*}{30 dias ou mais } & 69 & 50,7 & 136 & 55,5 & 1,0 \\
\hline & & & & & $(p=0,430)$ \\
\hline \multicolumn{6}{|l|}{$B C G$} \\
\hline Não & 34 & 25,2 & 65 & 27,1 & $0,91(0,54-1,51)$ \\
\hline \multirow[t]{2}{*}{ Sim } & 101 & 74,8 & 175 & 72,9 & 1,0 \\
\hline & & & & & $(p=0,781)$ \\
\hline \multicolumn{6}{|l|}{ Internação prévia } \\
\hline Sim & 57 & 41,9 & 131 & 53,0 & $0,64(0,45-1,00)$ \\
\hline \multirow[t]{2}{*}{ Não } & 79 & 58,1 & 116 & 47,0 & 1,0 \\
\hline & & & & & $(p=0,048)$ \\
\hline \multicolumn{6}{|l|}{ Diarréia } \\
\hline Um ou mais episódios & 45 & 34,6 & 104 & 42,6 & $0,71(0,45-1,13)$ \\
\hline \multirow[t]{2}{*}{ Nenhum episódio } & 85 & 65,4 & 140 & 57,4 & 1,0 \\
\hline & & & & & $(p=0,163)$ \\
\hline \multicolumn{6}{|l|}{ Desidratação } \\
\hline Sim & 31 & 23,8 & 89 & 36,3 & $0,55(0,33-0,90)$ \\
\hline \multirow[t]{2}{*}{ Não } & 99 & 76,2 & 155 & 63,7 & 1,0 \\
\hline & & & & & $(p=0,018)$ \\
\hline \multicolumn{6}{|l|}{ Gripe/Resfriado } \\
\hline Um ou mais episódios & 75 & 59,1 & 146 & 61,1 & $0,92(0,58-1,46)$ \\
\hline \multirow[t]{2}{*}{ Nenhum episódio } & 52 & 40,9 & 93 & 38,9 & 1,0 \\
\hline & & & & & $(p=0,790)$ \\
\hline \multicolumn{6}{|l|}{ Pneumonia prévia } \\
\hline Sim & 29 & 22,1 & 76 & 31,1 & $0,63(0,37-1,06)$ \\
\hline \multirow[t]{2}{*}{ Não } & 102 & 77,9 & 168 & 68,9 & 1,0 \\
\hline & & & & & $(p=0,083)$ \\
\hline \multicolumn{6}{|l|}{ Antecedente/Alergias } \\
\hline Sim & 31 & 23,5 & 43 & 18,1 & $1,39(0,80-2,42)$ \\
\hline \multirow[t]{2}{*}{ Não } & 101 & 76,5 & 195 & 81,9 & 1,0 \\
\hline & & & & & $(p=0,266)$ \\
\hline \multicolumn{6}{|l|}{ Serviços procurados* } \\
\hline Até dois & 86 & 63,2 & 100 & 40,5 & $2,53(1,61-3,99)$ \\
\hline \multirow[t]{2}{*}{ Mais de dois } & 50 & 36,8 & 147 & 59,5 & 1,0 \\
\hline & & & & & $(p<0,001)$ \\
\hline
\end{tabular}

* Nos 15 dias antecedentes à data do óbito 
Em ambos os grupos, as mães ou responsáveis declararam ter procurado serviços médicos nos últimos 15 dias antecedentes ao óbito. Frequientemente houve mais de uma visita aos serviços de saúde. Para o grupo dos óbitos em domicílio, 11 crianças não haviam sido levadas a nenhum serviço de saúde nos últimos 15 dias de vida. Para esse grupo observou-se uma média de 1,9 visitas aos serviços de saúde. Algumas mães relataram o óbito poucas horas após a avaliação e liberação médica "para casa" e 29 mães referiram o óbito até seis dias após a alta hospitalar. No grupo dos óbitos hospitalares a média para os últimos 15 dias foi de 2,8 visitas a serviços de saúde. O tipo de serviço mais procurado para ambos os grupos foi semelhante: serviços de pronto-socorro de hospitais públicos ou de hospitais privados conveniados com o serviço público de saúde $(79,1 \%$ dos óbitos domiciliares e $81,7 \%$ dos óbitos hospitalares), centros de saúde (17\% dos óbitos domiciliares e $14,8 \%$ dos óbitos hospitalares) e outros serviços, tais como clínicas e consultórios (3,9\% dos óbitos domiciliares e 3,5\% dos óbitos hospitalares).

A Tabela 4 refere-se aos atendimentos em serviços de saúde ocorridos nos últimos 15 dias de vida. O grupo de óbitos domiciliares realizou, nesse período, 259 visitas (média de 1,9), enquanto o grupo dos óbitos hospitalares realizou 689 visitas (média de 2,8). Entre as variáveis estudadas, a conduta classificada como "inadequada" mostrou-se significante associada ao óbito domiciliar.

Tabela 4

Análise da associação entre variáveis relativas aos atendimentos realizados para a patologia que levou ao óbito e local de ocorrência do óbito. Região Metropolitana de Belo Horizonte, MG, Brasil, de 1991 a 1992.

\begin{tabular}{|c|c|c|c|c|c|}
\hline \multirow{2}{*}{ Variáveis } & \multicolumn{2}{|c|}{ Óbitos domiciliares } & \multicolumn{2}{|c|}{ Óbitos hospitalares } & \multirow{2}{*}{ OR (IC95\%) } \\
\hline & $\mathrm{n}$ & $\%$ & $\mathrm{n}$ & $\%$ & \\
\hline \multicolumn{6}{|l|}{ Intervalo/consultas* } \\
\hline Três ou mais dias & 78 & 30,1 & 219 & 34,4 & $0,92(0,67-1,28)$ \\
\hline Até dois dias & 181 & 69,9 & 470 & 65,6 & $\begin{array}{l}1,0 \\
(p=0,678)\end{array}$ \\
\hline \multicolumn{6}{|l|}{ Atendimento } \\
\hline Outros profissionais & 33 & 12,7 & 43 & 6,2 & $1,60(0,97-2,65) * *$ \\
\hline Médicos & 226 & 87,3 & 646 & 93,8 & $\begin{array}{l}1,0 \\
(p=0,067)\end{array}$ \\
\hline \multicolumn{6}{|l|}{ Conduta } \\
\hline Inadequada & 148 & 57,1 & 281 & 40,8 & $1,78(1,31-2,41)^{* * *}$ \\
\hline Adequada & 111 & 42,9 & 408 & 59,2 & $\begin{array}{l}1,0 \\
(p<0,001)\end{array}$ \\
\hline \multicolumn{6}{|l|}{ Aderência à prescrição } \\
\hline Não & 62 & 2,3 & 10 & 1,5 & $1,61(0,48-4,95)$ \\
\hline Sim & 53 & 97,7 & 679 & 98,8 & $\begin{array}{l}1,0 \\
(p=0,397)\end{array}$ \\
\hline
\end{tabular}

* Quando houve mais de uma visita a serviços de saúde; ** OR ajustado para o tipo de profissional que realizou o atendimento; *** OR ajustado para o tipo de conduta.

Considerando o fato de que duas variáveis apresentadas nesta tabela poderiam apresentar-se como fatores de confusão entre si, foi realizado ajustamento através do método de Mantel-Haenzel para ambas. Assim, o atendimento foi ajustado para o tipo de conduta e essa foi ajustada para o tipo de profissional que prestou o atendimento. 


\section{Discussão}

O presente trabalho apresenta algumas limitações que devem ser levadas em consideração durante a sua análise. A primeira delas diz respeito à época de ocorrência dos óbitos. Atualmente, quase dez anos após a coleta dos dados, provavelmente a realidade do País não é a mesma. Entretanto a estrutura de mortalidade infantil tem ainda uma considerável proporção de óbitos concentrados no período pósneonatal.16 A rede pública de saúde ainda busca os melhores caminhos para a universalização e equidade da assistência. Por outro lado, as estatísticas oficiais ainda não refletem a realidade de modo fidedigno e seu elevado custo presume um progresso modesto para o Brasil e para a maioria dos países em desenvolvimento. ${ }^{3}$ Assim, admitindo-se que, infelizmente, a ocorrência de óbitos no domicílio ainda seja uma realidade, a sua análise representa um compromisso ético com a sociedade.

É importante considerar ainda o critério para definição do local do óbito como outra possível limitação desse estudo. Embora sejam bastante precisos os critérios de registro na declaração de óbito e de registro nos prontuários hospitalares, pode-se discutir a relevância da história materna declarando o óbito como extra-hospitalar (em domicílio ou a caminho do serviço de saúde), já que a família nem sempre está apta para reconhecer o óbito. Esse critério, porém, só foi utilizado, quando, além do relato da mãe, não se encontrava nenhum registro de internação hospitalar da criança após duas tentativas por entrevistadores diferentes. Pode-se também questionar o fato de que crianças falecidas em setor de urgência ou até duas horas após a hospitalização (outros critérios) poderiam estar sendo efetivamente assistidas. Contudo deve-se salientar o fato de que esse seria um atendimento tardio e que uma intervenção precoce e oportuna poderia, em um momento anterior, ter levado a outro desfecho.

Por outro lado, o presente estudo tem o mérito de disponibilizar uma fonte extra e confiável sobre as condições de acesso e qualidade dos serviços de saúde. Também propicia subsídios para uma avaliação crítica das estatísticas oficiais e disponibiliza dados para estudos posteriores. O enfoque específico sobre óbitos pós-neonatais evitáveis de ocorrência domiciliar é relativamente escasso na literatura nacional. Os significativos decréscimos na mortalidade infantil nos últimos anos, particularmente no seu componente pós-neonatal, talvez justifiquem esse fato. É importante ressaltar, porém, que apesar dos índices decrescentes, o Brasil ainda tem estimativas bem superiores aos países desenvolvidos e mesmo em relação a alguns países vizinhos. 16

Os resultados apresentados neste estudo são semelhantes aos resultados de trabalhos anteriores que também constataram que uma significativa proporção de óbitos infantis ocorrem fora do ambiente hospitalar.8,10,11 Análises anteriores também destacaram a deficiência qualitativa dos serviços de saúde, mostrando que algumas crianças morrem em casa após terem sido avaliadas em diversos serviços médicos. ${ }^{8}$ De forma similar, o presente estudo aponta a impropriedade de se avaliarem os serviços de saúde apenas em relação ao número de atendimentos realizados, sem a devida ponderação sobre a qualidade e a efetividade do atendimento realizado.

Um importante declínio das taxas de mortalidade infantil em países em desenvolvimento, de modo geral, sem uma significativa melhoria da qualidade de vida em tais países, destaca o papel dos serviços e das medidas de saúde pública para a sobrevida infantil. 17,18 Mas a persistência do complexo desnutriçãodiarréia-pneumonia como um importante grupamento de causas na determinação do óbito infantil, remete à idéia da evitabilidade e da responsabilidade dos serviços de saúde. 6 O conceito de morte infantil "evitável" não é recente e implica na disponibilidade de serviços de saúde eficazes, de boa qualidade. 19 Assume-se que serviços de saúde, em seus diversos níveis, ainda que não sejam capazes de interferir completamente no processo de causalidade da doença, deveriam ser aptos a reconhecê-la e tratá-la adequadamente. Ao destacar a mortalidade infantil por causas evitáveis, o presente trabalho discute o redimensionamento dos determinantes do óbito infantil. Particularizando o estudo sobre os óbitos em domicílio, procurou-se enfatizar a questão do acesso, como importante componente da qualidade da assistência.

A proposta de utilização da proporção de óbitos em domicílio como um indicador da atuação dos serviços de saúde não representa novidade. Entretanto, conforme salientam Duchiade et al., 8 as estatísticas oficiais não são capazes de identificar a verdadeira magnitude do problema. Segundo essas autoras, o que provavelmente ocorre é que o médico que atesta o óbito declara o local de ocorrência como sendo o próprio hospital, procurando poupar a família da desgastante passagem por um serviço de medicina legal. Agindo assim, entretanto, o médico negligencia a geração de dados estatísticos fidedignos e o local de ocorrência do óbito deixa de ser um indicador de acesso ou qualidade dos serviços de saúde para as estatísticas oficiais. Essa situação parece não ser peculiar do Brasil.20 Reafirma-se, então, a necessidade de maior conscientização para a 
classe médica da responsabilidade sobre a geração de dados estatísticos.

Em princípio, seria pouco provável que o acesso aos serviços de saúde representasse para a RMBH uma questão tão crítica, já que existia desde à época de coleta dos dados, uma grande rede ambulatorial e hospitalar. Um gradiente socioeconômico entre os dois grupos estudados poderia explicar a questão do acesso diferencial. Entretanto, os resultados deste estudo indicam que os grupos analisados eram uniformes e socialmente menos favorecidos, o que está de acordo com estudo prévio no sul do país, relativo às mortes infantis por pneumonia. $11 \mathrm{~A}$ elevada proporção de moradores em favelas, residentes em habitações precárias, com poucos eletrodomésticos, não servidos por uma rede de esgoto ou não dispondo de serviço sanitário satisfatório, já destaca uma população cujo acesso aos bens de consumo e serviços em geral é bastante limitado. Essa homogeneidade entre os dois grupos certamente é decorrente da reunião de causas investigadas, que ocorrem prioritariamente em estratos sociais de baixa renda. Embora as pneumonias ocorram em todas as classes sociais, os óbitos são mais freqüentes entre as crianças pobres. Para as doenças diarréicas e para a desnutrição, tanto a incidência como a mortalidade são maiores entre os mais pobres. Esse fato poderia estar facilitando a identificação de variáveis como o acesso e a qualidade da assistência médica. Todavia, as variáveis relativas aos serviços de saúde também são consideradas como determinantes do óbito infantil, independentemente do modelo adotado priorizar ou não o nível socioeconômico. 12

Os resultados também indicam que as crianças que faleceram prematuramente tiveram condições de gestação e parto similares e a proporção elevada de mães que não chegaram a realizar um número mínimo de consultas pré-natais reforça a questão do acesso. Uma proporção igualmente elevada de crianças nascidas de baixo peso pode também alertar para uma qualidade deficiente do atendimento pré-natal, incapaz de interferir com as condições sanitárias e/ou obstétricas precárias.

É importante registrar ainda que a acessibilidade não implica apenas na disponibilidade geográfica dos serviços, mas no fato de que os serviços sejam utilizados por quem realmente necessite. ${ }^{21}$ Implícito no conceito de acesso está também a aceitabilidade dos serviços, o que depende da valorização por parte do usuário. 22 A observação de que os serviços mais procurados foram pronto-socorros traduz a percepção de maior resolutividade destes serviços para as famílias e/ou uma baixa credibilidade dos postos de cuidados primários (ambulatórios e centros de saúde). Todavia, os pronto-socorros quase sempre realizam um atendimento pontual, sem compromisso com a rede de atenção primária. Talvez esse atendimento desarticulado seja o ponto mais crítico da interface entre cuidados primários e secundários. Não existindo compromisso com a integralidade da assistência, o atendimento de pronto-socorro limita-se à queixa do paciente e, freqüentemente, a subestima. A hospitalização não representa por si só um evento desejável ou que otimize os cuidados médicos, particularmente para as doenças aqui estudadas. Porém, representa um cuidado fundamental para a assistência de lactentes gravemente enfermos. Sob esse aspecto, para as crianças estudadas, ter uma chance maior de ser hospitalizada dependeu muito mais da perseverança da família, através de uma busca repetitiva aos serviços de saúde, do que do reconhecimento do próprio serviço de saúde das necessidades da criança e da família.

Pode-se questionar o fato de que buscas repetidas aos serviços de saúde sejam um indicador de maior gravidade do quadro. As variáveis associadas com o óbito hospitalar na análise univariada poderiam estar sinalizando esse fato. Todavia, essa maior gravidade não foi reconhecida oportunamente pelos serviços de saúde procurados, o que depõe contra a qualidade dos mesmos: um atendimento oportuno e criterioso poderia ter levado a outro desfecho.

A análise específica das visitas aos serviços de saúde realizadas pelos dois grupos destacou a conduta inadequada como determinante do óbito extrahospitalar. Permitiu inferir também que a família quase sempre reconheceu a necessidade de intervenção médica. A elevada proporção de aderência às prescrições em ambos os grupos indica aceitação e confiança por parte dos familiares. Por outro lado, em ambos os grupos, mais de $65 \%$ das famílias voltaram a buscar o serviço de saúde em até dois dias após o atendimento. Esse fato, diante da contundente evolução dos casos, revela uma baixa resolutividade e uma qualidade crítica na assistência, particularmente se observarmos que muitas crianças chegaram a passar por até dez serviços de saúde antes de morrer ou evoluíram para o óbito poucas horas após consulta e liberação médica.

Frente aos resultados deste estudo, parece incontestável a necessidade de reorganização das práticas de saúde de modo geral, com ênfase na credibilidade, resolutividade e aceitabilidade dos serviços de cuidados primários e sua interface com os serviços hospitalares. Estudos anteriores já destacavam a necessidade de avaliações estruturais, administrativas e qualitativas nos serviços de saúde oferecido à população em geral, a partir da análise das condições do 
óbito infantil.5,8,11,23 Discutir a questão da mortalidade infantil nesse contexto não representa reducionismo dos determinantes socioeconômicos,

\section{Agradecimentos}

Os autores agradecem as entidades que propiciaram o financiamento para este estudo: Organização Panamericana de Saúde (OPAS) e Fundação do Amparo à Pesquisa de Minas Gerais (FAPEMIG).

\section{Referências}

1. OPS (Organização Panamericana de la Salud). Las condiciones de salud en las Americas. Washington, DC: OPS; 1990. (Publicación científica, 524).

2. Puffer RR, Serrano CV. Patterns of mortality in childhood. Washington, DC: PAHO (Pan American Health Organization; 1973. (Scientific Publication, 262).

3. Ahmad OB, Lopez AD, Inoue M. The decline in child mortality: a reappraisal. Bull World Health Organ 2000 78:1175-91

4. Rutstein SO. Factors associated with trends in infant and child mortality in developing countries during the 1990s. Bull World Health Organ 2000; 78:1256-70.

5. Formigli VLA, Silva LMV, Cerdeira AJP, Pinto CME Oliveira RSA, Caldas AC, Vilas Boas MJB, Fonseca AC, Souza LSF, Silva LR, Paes MSF. Avaliação da atenção à saúde através da investigação de óbitos infantis. Cad Saúde Publica 1996; 12: 33-41.

6. Hartz ZMA, Champagne F, Leal MC, Contandriopoulos AP. Mortalidade infantil "evitável" em duas cidades do Nordeste do Brasil: indicador de qualidade do sistema de saúde local. Rev Saúde Pública 1996; 30: 310-8.

7. Barros FC, Victora CG, Teixeira AMB, Puerto Filho M. Mortalidade perinatal e infantil em Pelotas, Rio Grande do Sul: nossas estatísticas são confiáveis? Cad Saúde Pública 1985; 1: 348-58.

8. Duchiade MP, Carvalho ML, Leal, MC. As mortes "em domicílio" de menores de um ano na região metropolitana do Rio de Janeiro em 1986 - um "evento - sentinela" na avaliação dos serviços de saúde. Cad Saúde Pública 1989; 5: 251-63.

9. Mendonça EF, Goulart EMA, Machado JAD. Confiabilidade da declaração de causa básica de mortes infantis em região metropolitana do sudeste do Brasil. Rev Saúde Pública 1994; 28: 385-91.

10. Menezes AMB, Victora CG, Barros FC, Menezes FS, Jannke H, Albernaz, Menezes FS, Jannke HA, Alves C, Rocha C. Estudo populacional de investigação de óbitos mas antes, um compromisso com a e equidade do acesso e com a qualidade dos resultados em todos os níveis de assistência à saúde infantil. perinatais e infantis: metodologia, validade do diagnóstico e sub-registro. J Pediatr [Rio de Janeiro] 1997; 73 : 383-7.

11. Chatkin JM, Braga JU, Procópio MJ, Dalcomo MP. Estudo de óbitos infantis por pneumonia por meio de inquérito domiciliar. J Pediatr [Rio de Janeiro] 1992; 68: 116-22.

12. França E. Estudo de determinantes da mortalidade infantil por diarréia, pneumonia e desnutrição na Região Metropolitana de Belo Horizonte em 1991-1992 [tese doutorado]. Belo Horizonte: Departamento de Medicina Tropical, Faculdade de Medicina da Universidade Federal de Minas Gerais; 1995

13. França E, Souza JM, Guimarães MDC, Goulart EMA, Colosimo E, Antunes CMF. Associação entre fatores socioeconômicos e mortalidade infantil por diarréia, pneumonia e desnutrição em região metropolitana do Sudeste do Brasil: um estudo caso-controle. Cad Saúde Pública 2001; 17: 1437-47.

14. Dean AG, Burton AH, Dicker RC. Epi-info: a word processing, database and statistics program for epidemiology [computer program]. Version 2. Atlanta: Center for Disease Control; 1994.

15. Campos-Filho N, Franco EL. A microcomputer program for multiple logistic regression by unconditional and conditional maximum likelyhood methods. Am J Epidemiol 1989; 129: 439-44.

16. Szwarcwald CL, Leal MA, Castilho EA, Andrade CLT. Mortalidade infantil no Brasil: Belíndia ou Bulgária? Cad Saúde Pública 1997; 13: 503-16.

17. Claeson M, Bos ER, Mawi T, Pathmanathan I. Reducing child mortality in India in the new millennium. Bull World Health Organ 2000; 78: 1192-9.

18. Victora CG, Barros FC. Repensando a associação entre indicadores de saúde e de qualidade de vida. In: Costa MFFL, Sousa RP, organizadores. Qualidade de vida: compromisso histórico da epidemiologia. Belo Horizonte: Coopmed (Cooperativa Médica Editora); 1994. p. 19-23. 
19. Rutstein DD, Berenberg W, Chalmers TC, Child CG, Fishman AP, Perrin EB. Measuring the quality of medical care: a clinical method. N Engl J Med 1976; 294: 582-8.

20. Rodríguez H, Medero D, Rossello JL, Ferrari AM. Muerte em domicilio em el período posneonatal: Montevideo, 1996. Rev Med Uruguay 1998; 14: 147-53.

Recebido em 28 de maio de 2002

Versão final reapresentada em 20 de julho de 2002

Aprovado em 26 de agosto de 2002
21. Aday LA, Andersen R. A framework for the use of acces to medical care. Health Serv Res 1974; 9: 208-20

22. Donabedian A. The seven pillars of quality. Arch Pathol Lab Med 1990; 114: 1115-8.

23. Caldeira AP, França E, Goulart EMA. Mortalidade infantil pós-neonatal e qualidade da assistência médica: um estudo caso-controle. J Pediatr [Rio de Janeiro] 2001; 77: 461-8. 